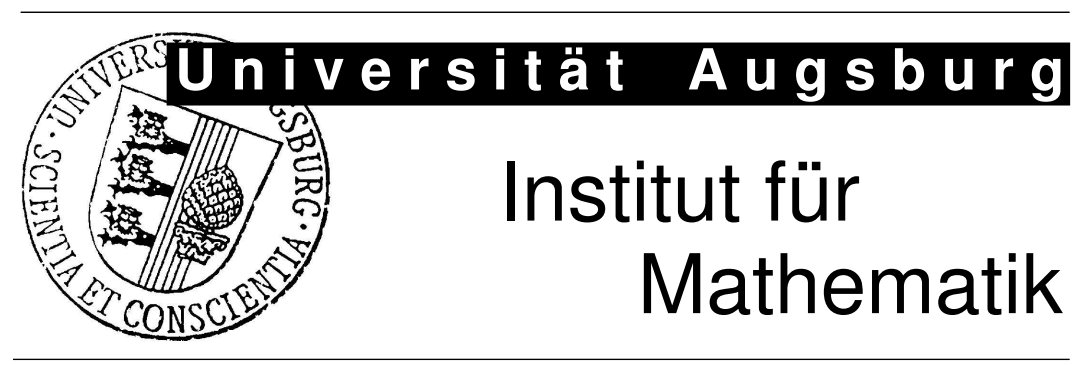

Ronald H.W. Hoppe, Christopher Linsenmann, Kidist Zeleke

Numerical Simulation of Surface Acoustic Wave Actuated Enantiomer Separation by the Finite Element Immersed Boundary Method 


\section{Impressum:}

\section{Herausgeber:}

Institut für Mathematik

Universität Augsburg

86135 Augsburg

http://www . math. uni-augsburg.de/de/forschung/preprints.html

\section{ViSdP:}

Ronald H.W. Hoppe

Institut für Mathematik

Universität Augsburg

86135 Augsburg

Preprint: Sämtliche Rechte verbleiben den Autoren (C) 2012 


\title{
NUMERICAL SIMULATION OF SURFACE ACOUSTIC WAVE ACTUATED ENANTIOMER SEPARATION BY THE FINITE ELEMENT IMMERSED BOUNDARY METHOD
}

\author{
R.H.W. HOPPE* , C. LINSENMANN ${ }^{\dagger}$, AND K. ZELEKE ${ }^{\ddagger}$
}

\begin{abstract}
Enantiomers are chiral objects such as chemical molecules that can be distinguished by their handedness. They typically occur as racemic compounds of left- and right-handed species which may have completely different properties. Therefore, in applications such as drug design in pharmacology, enantiomer separation is an important issue. Here, we present a new technology for enantiomer separation by surface acoustic wave generated vorticity patterns consisting of pairwise counter-rotating vortices in a carrier fluid. The enantiomers are injected onto the surface of the fluid between two counter-rotating vortices such that right-handed (left-handed) enantiomers get attracted by left-rotating (right-rotating) vortices. In particular, we are concerned with the numerical simulation of this separation process by an application of the finite element immersed boundary method which relies on the solution of a coupled system consisting of the incompressible NavierStokes equations and the equations of motion of the immersed enantiomers described with respect to an Eulerian and a Lagrangian coordinate system. For a model system of deformable, initially L-shaped enantiomers the results of the numerical simulations reveal a perfect separation.
\end{abstract}

Key words. enantiomer separation, surface acoustic waves, finite element immersed boundary method

AMS subject classifications. 65M60, 74L15, 76Z05, 92C10, 92C50

1. Introduction. A geometric object is said to be chiral, if it is not identical to its mirror image, and achiral, otherwise. A chiral object and its mirror image are called enantiomers (or optical isomers). Since the word chiral stems from the Greek ' $\chi \varepsilon\llcorner\rho$ ' which means 'hand', one distinguishes enantiomers by their handedness (rightresp. left-handedness, or R- resp. L-form, or (+)- resp. (-)-form).
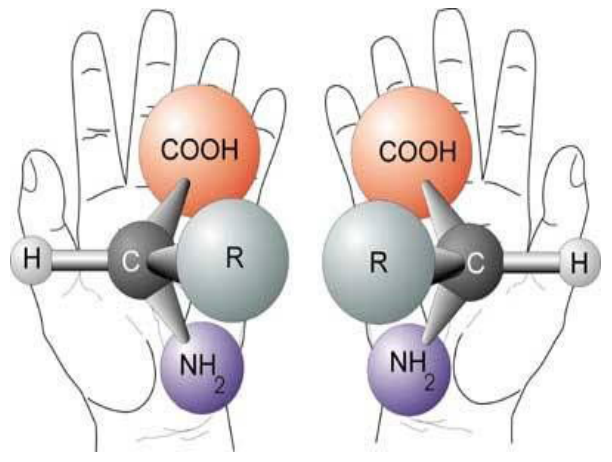

FIG. 1.1. Left- and right-handed enantiomer

*Institute of Mathematics, University of Augsburg, D-86159, Augsburg, Germany, and Department of Mathematics, University of Houston, Houston, TX 77204-3008, USA (rohop@math.uh.edu). The author has been supported by the DFG Priority Programs SPP 1253 and SPP 1506, by the NSF grants DMS-0914788, DMS-1115658, and by the European Science Foundation within the Networking Programme 'OPTPDE'.

${ }^{\dagger}$ Institute of Mathematics, University of Augsburg, D-86159, Augsburg, Germany. The author has been supported by the DFG Priority Program SPP 1253.

${ }_{\ddagger}^{\ddagger}$ Department of Mathematics, University of Houston, Houston, TX 77204-3008, USA. The author has been supported by the NSF grant DMS-1115658. 
In chemistry, chirality refers to a molecule that is not superposable on its mirror image (cf. Figure 1.1). Compounds consisting of molecules of the same handedness are called single-enantiomeric, enantiopure, or unichiral, whereas compounds consisting of the same amount of R- and L-form enantiomers are referred to as racemic. The discovery of molecular chirality goes back to the nineteenth century when in 1815 the French physicist J.-B. Biot [3] studied organic compounds and found that some of them rotate polarized light in the noncrystalline state, i.e., in the liquid or solvent state. Biot was aware that the optical rotation is due to structural properties of the molecules, and he referred to them as 'substances moléculairement actives'. Three decades later, in 1848 the French chemist and microbiologist Louis Pasteur [27] (see also [15]) discovered hemihedrism in crystals of dextro-tartaric acid ((+)-tartaric acid), i.e., the existence of small facets at alternate corners of the crystals that make the crystals dissymmetric (i.e., chiral). Pasteur [28] also examined racemic acid, earlier discovered by Gay-Lussac in 1826 which does not rotate polarized light and is chemically inactive, and found that there are two different crystals with hemihedral facets inclined to the right and to the left. Pasteur managed to separate the two kind of crystals $((+)-$ and $(-)$-tartaric acid) and found that in solvent state they rotate polarized light with the rotations being equal in magnitude but opposite in direction. In 1858, Pasteur also discovered enantiomer selectivity when he studied solutions of racemic acid enriched by microorganisms and found that the $(+)$-enantiomers were more rapidly metabolized than the (-)-enantiomers concluding that this process must be due to a selective interaction of enantiomers with key chiral molecules within the microorganisms. Roughly twenty years after Pasteur's fundamental work, the Dutch and French chemists J.H. van't Hoff [17] and J.A. LeBel [24] independently discovered the tetrahedral carbon atom as a basis for molecular chirality and thus paved the way for the elucidation of the structures of organic compounds.

During the first half of the last century, several attempts have been made to derive appropriate models for chiral molecules based on electronic theories explaining their optical activity. Among them are Born's theory of coupled oscillators [6] and the quantum mechanical one-electron theory due to Condon et al. [10] which - opposed to Born's assumption - proves that a single electron can be optically active under the influence of a chiral potential. However, the quantum mechanical description of chirality leads to a contradiction which already has been stated ten years before Condon's contribution by Hund [19] known as Hund's paradox: stable ground energy states of chiral molecules with respect to a two-well potential are achiral, whereas the L- and R-states formed by eigenstates associated with the two local minima are not stationary and can tunnel through the potential barrier such that an enantiomer should permanently switch between its L- and R-form. Obviously, there must be an additional coupling effect which destabilizes the achiral ground state of quantum mechanics and stabilizes the L- and R-form once the molecule has been synthesized accordingly. A possible effect is provided by electro-weak quantum chemistry: thirty years ago, computations revealed that there is an energy difference between L- and R-form enantiomers in achiral media ('parity violation' resp. 'de lege symmetry breaking') which dominates the tunneling effect. This result is of significant relevance for the understanding of molecular chirality: only in case of parity violation the R- and L-form of enantiomers can be observed in an absolute sense (cf., e.g., [32]). The current status of spectroscopic experiments, confirming molecular parity violation, is reviewed in [33].

Nowadays, enantiomer separation and enantiomer selectivity play a significant role 
in agrochemical, electronic, and pharmaceutical as well as food, flavor and fragrance industries (cf., e.g., $[2,8,9,13,23,29]$ ). The relevance of chirality in drug design became apparent on occasion of the so-called 'Contergan scandal' or 'thalidomide disaster' in the sixties of the last century when worldwide thousands of children were born with extremely severe deformities after their mothers had taken this sleeping drug in early pregnancy. Unfortunately, it was discovered too late that L-thalidomide molecules cause malformations of the fetus, since they block the action of a chiral enzyme regulating the synthesis of cartilage in the second month of the pregnancy.

The qualitative and quantitative analysis of chiral molecules relies on high-throughput screening by fluorescence spectroscopy and mass spectroscopy with achiral reporter molecules or antibodies and enzymes [7]. Since the chemical synthesis of enantiomers usually gives rise to racemic compounds, chiral separation is of utmost importance. Current approaches are based on direct gas chromatography [7] or HPLC ( Pressure Liquid Chromatography) [37], capillary electrophoresis [34], or NMR (Nuclear Magnetic Resonance) anisotropy methods [38]. They suffer from the drawbacks that they are slow and only yield endpoint results, i.e., they do not provide any information about the dynamics of the separation process. Moreover, they mostly require costly chiral media. Consequently, in order to guarantee a higher cost-effectiveness as well as a significant speed-up and to allow for an in-situ investigation of the enantiomer separation at a high time-resolution, there is the need for alternative techniques. Such techniques have been provided by chiral separation in microfluidic devices taking advantage of the fact that enantiomers drift in microflows with a direction depending on their chirality (cf., e.g., [7, 21, 22, 25, 26, 35]).
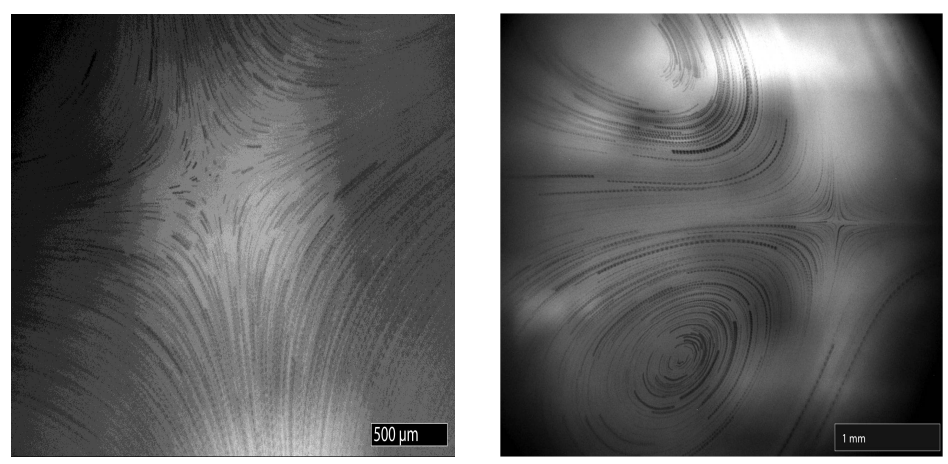

FIG. 1.2. Left: Vorticity pattern at the surface of the fluid. The optical path is slightly tilted to gain a larger field of view. The image is a superposition of micrographs and shows parts of the four quadrant flows induced by the SAWs. Right: Micrograph showing two of the counterrotating vortices. In the flow quadrant diagonally opposing vortices always have the same direction of rotation.

In this paper, we are concerned with the separation of deformable vesicle-like enantiomers by a specific flow pattern generated by surface acoustic waves (SAWs). In particular, we consider a fluid-filled container with an immersed SAW microchip at the ground. The SAW microchip is coated with a piezoelectric material such as lithium niobate $\left(\mathrm{LiNbO}_{3}\right)$ and features an Inter-Digital Transducer (IDT) such that the IDT is placed at the center of the bottom of the container with its aperture pointing upwards. Applying a high-frequency signal to the IDT, acoustic waves are created that enter the fluid in the container and create a steady-state flow pattern at the fluid surface consisting of four counter-rotating vortices (cf. Figure 1.2). Almost flat L- 
shaped enantiomers with hydrophobic top and bottom are injected onto the surface of the fluid between two counter-rotating vortices. It turns out that right-handed (left-handed) enantiomers get trapped by left-rotating (right-rotating) vortices in the sense that they stably rotate around the center of the vortex.

For the numerical simulation of the surface acoustic wave actuated enantiomer separation we have used the Finite Element Immersed Boundary Method (FE-IB). The Immersed Boundary Method (IB) is due to Peskin [30] (cf. the survey paper [31] and the references therein) and has been extended to the FE-IB in $[4,5]$ (cf. also $[14,18])$. The FE-IB is based on a coupled system consisting of the incompressible Navier-Stokes equations described in an Eulerian coordinate system and the equation of motion of the immersed enantiomer described with respect to a Lagrangian coordinate system.

The paper is organized as follows: In section 2, we deal with the generation of vorticity patterns by SAWs. Section 3 is concerned with a description of the FE-IB, whereas section 4 is devoted to its numerical realization. Finally, section 5 contains the results of numerical simulations illustrating the feasibility of SAW actuated enantiomer separation.

2. Generation of the Vorticity Pattern by SAWs. The SAWs are generated by an IDT featuring fingers substantially parallel to one another. A static electric field $\mathbf{E}=-\nabla \Phi$, with $\Phi$ denoting the electric potential, is applied to generate a strain which varies across the aperture of the IDT. The electric field is created by applying a DC voltage between two correspondingly positioned conductors. The piezoelectric effect thus leads to SAWs that travel in the direction of the wall and enter the fluidfilled container.

We assume that the piezoelectric material with density $\rho$ occupies some domain $\Omega_{1}$ with boundary $\Gamma_{1}=\partial \Omega_{1}$ and exterior unit normal $\mathbf{n}_{1}$ such that

$$
\begin{aligned}
& \Gamma_{1}=\bar{\Gamma}_{E, D} \cup \bar{\Gamma}_{E, N} \quad, \quad \Gamma_{E, D} \cap \Gamma_{E, N}=\emptyset, \\
& \Gamma_{1}=\bar{\Gamma}_{p, D} \cup \bar{\Gamma}_{p, N} \quad, \quad \Gamma_{p, D} \cap \Gamma_{p, N}=\emptyset,
\end{aligned}
$$

where $\Gamma_{E, D}$ is a rectangular subdomain of the upper boundary of $\Gamma_{1}$ and $\Gamma_{E, N}:=$ $\Gamma_{1} \backslash \bar{\Gamma}_{E, D}$. Denoting by $\mathbf{u}$ the mechanical displacement, by $\boldsymbol{\sigma}=\boldsymbol{\sigma}(\mathbf{u}, \mathbf{E})$ the stress tensor, and by $\mathbf{D}=\mathbf{D}(\mathbf{u}, \mathbf{E})$ the dielectric displacement, and given boundary data $\Phi_{E, D}$ on $\Gamma_{E, D}$, the pair $(\mathbf{u}, \Phi)$ satisfies the following initial-boundary value problem for the piezoelectric equations (cf. [16])

$$
\begin{gathered}
\rho_{p} \frac{\partial^{2} \mathbf{u}}{\partial t^{2}}-\nabla \cdot \boldsymbol{\sigma}(\mathbf{u}, \mathbf{E})=0 \quad \text { in } Q_{1}:=\Omega_{1} \times\left(0, T_{1}\right), \\
\nabla \cdot \mathbf{D}(\mathbf{u}, \mathbf{E})=0 \quad \text { in } Q_{1} \\
\mathbf{u}=0 \quad \text { on } \Gamma_{p, D} \quad, \quad \mathbf{n}_{1} \cdot \boldsymbol{\sigma}=\boldsymbol{\sigma}_{\mathbf{n}_{1}} \quad \text { on } \Gamma_{p, N} \\
\Phi=\Phi_{E, D} \quad \text { on } \Gamma_{E, D} \quad, \quad \mathbf{n}_{1} \cdot \mathbf{D}=D_{\mathbf{n}_{1}} \quad \text { on } \Gamma_{E, N} \\
\mathbf{u}(\cdot, 0)=0 \quad, \quad \frac{\partial \mathbf{u}}{\partial t}(\cdot, 0)=0 \quad \text { in } \Omega_{1} .
\end{gathered}
$$

The equations have to be complemented by the generalized Hooke's law

$$
\boldsymbol{\sigma}(\mathbf{u}, \mathbf{E})=\mathbf{c} \varepsilon(\mathbf{u})-\mathbf{e} \mathbf{E},
$$

and the constitutive equations

$$
\mathbf{D}=\epsilon \mathbf{E}+\mathbf{P}, \quad \mathbf{P}=\mathbf{e} \varepsilon(\mathbf{u})
$$


Here, $\varepsilon(\mathbf{u}):=\left(\nabla \mathbf{u}+(\nabla \mathbf{u})^{T}\right) / 2$ is the linearized strain tensor and $\mathbf{c}, \mathbf{e}$ refer to the symmetric fourth order elasticity tensor and the symmetric third order piezoelectric tensor. Moreover, $\boldsymbol{\epsilon}$ is the electric permittivity of the material and $\mathbf{P}$ stands for the polarization.

We assume that the fluid in the container occupies a domain $\Omega_{2}:=(0, L)^{2} \times(0, H), H$, $L>0$, with boundary $\Gamma_{2}:=\Gamma_{b} \cup \Gamma_{l} \cup \Gamma_{s}$, where $\Gamma_{b}:=(0, L) \times(0, L) \times\{0\}, \Gamma_{s}:=$ $(0, L) \times(0, L) \times\{H\}$, and $\Gamma_{l}:=\Gamma_{2} \backslash\left(\Gamma_{b} \cup \Gamma_{s}\right)$. The impact of the SAWs on the fluid flow is modeled through a boundary condition on $\Gamma_{S A W}:=[L / 2-a, L / 2+$ $a] \times\{0\}, a>0$. Taking into account that the propagation of acoustic waves in fluids yields compressible effects and denoting by $\rho_{f}, \eta, \xi$ the density, the standard and the bulk viscosity of the fluid, and by $\mathbf{v}, p$ the velocity and the pressure, the fluid flow is described by the compressible Navier-Stokes equations

$$
\begin{aligned}
\rho_{f}\left(\frac{\partial \mathbf{v}}{\partial t}+\mathbf{v} \cdot \nabla \mathbf{v}\right) & =\nabla \cdot \boldsymbol{\sigma} \\
\frac{\partial \rho_{f}}{\partial t}+\nabla \cdot\left(\rho_{f} \mathbf{v}\right) & =0 \quad \text { in } Q_{2}:=\Omega_{2} \times\left(0, T_{2}\right), \\
\mathbf{v}(\cdot, t) & =\frac{\partial \mathbf{u}}{\partial t}(\cdot, t) \quad \text { on } \Gamma_{S A W}, t \in\left(0, T_{2}\right), \\
\mathbf{v}(\cdot, t) & =\mathbf{0} \quad \text { on }\left(\Gamma_{b} \backslash \Gamma_{S A W}\right) \cup \Gamma_{l}, t \in\left(0, T_{2}\right), \\
\boldsymbol{\sigma} \mathbf{n} & =\mathbf{0} \quad \text { on } \Gamma_{s}, t \in\left(0, T_{2}\right), \\
\mathbf{v}(\cdot, 0)=\mathbf{0}, & p(\cdot, 0)=0 \quad \text { in } \Omega_{2} .
\end{aligned}
$$

The constitutive equation for the stress tensor $\boldsymbol{\sigma}=\left(\sigma_{i j}\right)_{i, j=1}^{3}$ reads

$$
\sigma_{i j}:=-p \delta_{i j}+2 \eta D_{i j}(\mathbf{v})+\delta_{i j}(\xi-2 \eta / 3) \nabla \cdot \mathbf{v},
$$

where $\mathbf{D}=\left(D_{i j}\right)_{i, j=1}^{3}, D_{i j}:=\left(\partial v_{i} / \partial x_{j}+\partial v_{j} / \partial x_{i}\right) / 2$ stands for the rate of deformation tensor.

The SAW induced fluid flow exhibits two different time scales. When the SAWs enter the fluid filled container, sharp jets are created within nanoseconds. The SAWs propagate upwards and experience a significant damping which results in a vorticity flow pattern called acoustic streaming. This relaxation process happens on a time scale of milliseconds. The multiscale character of this process can be appropriately taken care of by a homogenization approach. We refer to [1, 20] for details.

3. The Finite Element Immersed Boundary Method. In this section, we adopt standard notation from Lebesgue and Sobolev space theory (cf., e.g., [36]). In particular, for $D \subset \mathbb{R}^{2}$ we refer to $L^{2}(D)$ and $H^{s}(D)$ as the Hilbert space of Lebesgue integrable functions in $D$ with inner product $(\cdot, \cdot)_{0, D}$ and associated norm $\|\cdot\|_{0, D}$ and the Sobolev space of functions with inner product $(\cdot, \cdot)_{s, D}$ and norm $\|\cdot\|_{s, D} \cdot L_{0}^{2}(D)$ is the subspace of functions with zero integral mean. We further refer to $H_{0}^{1}(D)$ as the closure of $C_{0}^{\infty}(D)$ in $H^{1}(D)$ and to $H^{-1}(D)$ as the dual of $H_{0}^{1}(D)$, denoting by $\langle\cdot, \cdot\rangle_{H^{-1}, H_{0}^{1}}$ the dual pairing. For $\Sigma \subseteq \partial D$ and a function $v \in H^{s}(D)$, we denote by $\left.v\right|_{\Sigma}$ the trace of $v$ on $\Sigma$. We write $\mathbf{L}^{2}(D)$ and $\mathbf{H}^{s}(D)$ in case of vector-valued functions.

Since the enantiomers float on the surface $\Gamma_{s}$ of the fluid, we are only interested in the fluid flow and the motion of the enantiomers on $\Gamma_{s}$. The fluid flow on $\Gamma_{s}$ can be modeled by the incompressible Navier-Stokes equations with a source term $\mathbf{f}=\mathbf{f}_{q}+\mathbf{f}_{g}$ consisting of a quadrupolar force density $\mathbf{f}_{q}$, reflecting the SAW induced vorticity 
pattern on $\Gamma_{s}$, and a global force density $\mathbf{f}_{g}$, reflecting the impact of the enantiomers on the flow:

$$
\begin{array}{rlrl}
\rho_{f} \frac{\partial \mathbf{v}}{\partial t}+\rho(\mathbf{v} \cdot \nabla) \mathbf{v}-\eta \Delta \mathbf{v}+\nabla p & =\mathbf{f} & & \text { in } \Gamma_{s} \times(0, T), \\
\nabla \cdot \mathbf{v}=0 & & \text { in } \Gamma_{s} \times(0, T), \\
\mathbf{v}=\mathbf{0} & & \text { on } \partial \Gamma_{s} \times[0, T), \\
\mathbf{v}(\cdot, 0) & =\hat{\mathbf{v}} & & \text { in } \Gamma_{s} .
\end{array}
$$

The quadrupolar force density is given according to

$$
\mathbf{f}_{q}:=-\eta \Delta \hat{\mathbf{v}}, \hat{\mathbf{v}}=\left(\hat{v}_{1}, \hat{v}_{2}\right)^{T}, \hat{v}_{1}=\partial \Psi / \partial x_{1}, \hat{v}_{2}=-\partial \Psi / \partial x_{2}
$$

in terms of the stream function

$$
\Psi\left(x_{1}, x_{2}\right)=v_{0}(f) L \frac{\sin \left(\pi x_{1} / L\right) \sin \left(\pi x_{2} / L\right)}{\left(2-\cos \left(\pi x_{1} / L\right)\right)\left(2-\cos \left(\pi x_{2} / L\right)\right)},
$$

where $v_{0}(f)>0$ depends on the frequency $f$ of the IDT. We note that $\mathbf{f}_{q}$ provides a good approximation of the SAW generated vorticity pattern at the surface of the fluid. The global force density $\mathbf{f}_{g}$ will be specified by means of the total energy of the immersed boundary in (3.8) below.

In the FE-IB an immersed enantiomer is modeled as a body consisting of an elastic membrane enclosing a fluid which here is assumed to have the same density and viscosity as the carrier fluid in the container. In practice, this can be achieved by density and viscosity matching, i.e., adding chemical additives to the carrier fluid. The immersed enantiomer is supposed to occupy a subdomain $B_{t} \subset \Gamma_{s}, t \in[0, T]$, with boundary $\partial B_{t}$ which is a non-selfintersecting closed curve. We further assume that the boundary $\partial B_{0}$ of the initial configuration $B_{0}$ has length $\ell:=\left|\partial B_{0}\right|$ and denote by $q \in[0, \ell]$ the Lagrangian coordinate labeling a material point on $\partial B_{0}$. We refer to $\mathbf{X}(q, t)=\left(X_{1}(q, t), X_{2}(q, t)\right)^{T}$ as the position vector of the point $q$ at time $t \in(0, T]$ which moves with the velocity $\mathbf{v}$ of the fluid such that the equation of motion takes the form

$$
\begin{aligned}
\frac{d \mathbf{X}}{d t}(q, t) & =\mathbf{v}(\mathbf{X}(q, t), t), \quad q \in[0, \ell], t \in[0, T], \\
\mathbf{X}(q, 0) & =\mathbf{X}^{0}(q), \quad q \in[0, \ell]
\end{aligned}
$$

where $\mathbf{X}^{0}$ stands for the initial position.

The total elastic energy of the immersed boundary $\partial B_{t}$ of the enantiomer is given by

$$
\begin{aligned}
E(t) & :=E^{e}(t)+E^{b}(t), t \in(0, T), \\
E^{e}(t) & :=\int_{0}^{L} \mathcal{E}^{e}(\mathbf{X}(q, t)) d q, \quad E^{b}(t):=\int_{0}^{L} \mathcal{E}^{b}(\mathbf{X}(q, t)) d q,
\end{aligned}
$$

where $\mathcal{E}^{e}(t)$ and $\mathcal{E}^{b}(t)$ are the local energy densities according to

$$
\begin{aligned}
& \mathcal{E}^{e}(\mathbf{X}(q, t))=\frac{\kappa_{e}}{2}\left(\left|\frac{\partial \mathbf{X}}{\partial q}(q, t)\right|^{2}-1\right) \\
& \mathcal{E}^{b}(\mathbf{X}(q, t))=\frac{\kappa_{b}}{2}\left|\frac{\partial^{2} \mathbf{X}}{\partial q^{2}}(q, t)\right|^{2}
\end{aligned}
$$


Here, $\kappa_{e}>0$ and $\kappa_{b}>0$ denote the elasticity coefficients with respect to elongationcompression and bending.

Denoting by $\mathbf{f}_{l}$ the local force density according to $\mathbf{f}_{l}(q, t)=-E^{\prime}(\mathbf{X}(q, t))$, where $E^{\prime}$ is the Gâteaux derivative of $E$, the global force density $\mathbf{f}_{g}$ in (3.1a) is given in variational form by

$$
\left\langle\mathbf{f}_{g}(t), \mathbf{w}\right\rangle_{\mathbf{H}^{-1}, \mathbf{H}_{0}^{1}}=\int_{0}^{L} \mathbf{f}_{l}(q, t) \cdot \mathbf{w}(\mathbf{X}(q, t)) d q, \quad \mathbf{w} \in \mathbf{H}_{0}^{1}\left(\Gamma_{s}\right) .
$$

The FE-IB is based on the variational formulation of (3.1) and (3.4). To this end, we introduce the function spaces

$$
\begin{aligned}
\mathbf{V}(0, T) & :=\mathbf{H}^{1}\left((0, T), \mathbf{H}^{-1}\left(\Gamma_{s}\right)\right) \cap \mathbf{L}^{2}\left((0, T), \mathbf{H}^{1}\left(\Gamma_{s}\right)\right), \\
\mathbf{W}(0, T) & :=\left\{\mathbf{v} \in \mathbf{V}(0, T)|\mathbf{v}|_{\partial \Gamma_{s}}=\hat{\mathbf{v}}\right\}, \\
Q(0, T) & :=L^{2}\left((0, T), L_{0}^{2}\left(\Gamma_{s}\right)\right), \\
\mathbf{Y}(0, T) & :=\mathbf{H}^{1}\left((0, T), \mathbf{L}^{2}([0, \ell])\right) \cap \mathbf{L}^{2}\left((0, T), \mathbf{H}_{\text {per }}^{3}([0, \ell])\right),
\end{aligned}
$$

where $\mathbf{H}_{\text {per }}^{3}([0, \ell]):=\left\{\mathbf{Y} \in \mathbf{H}^{3}((0, \ell)) \mid \partial^{k} \mathbf{Y}(0) / \partial q^{k}=\partial^{k} \mathbf{Y}(\ell) / \partial q^{k}, k=0,1,2\right\}$.

The weak formulation of the Navier-Stokes equations (3.1) requires the computation of $(\mathbf{v}, p) \in \mathbf{W}(0, T) \times Q(0, T)$ such that for all $\mathbf{w} \in \mathbf{H}_{0}^{1}\left(\Gamma_{s}\right)$ and all $q \in L_{0}^{2}\left(\Gamma_{s}\right)$ there holds

$$
\begin{aligned}
\left\langle\rho \frac{\partial \mathbf{v}}{\partial t}, \mathbf{w}\right\rangle_{\mathbf{H}^{-1}, \mathbf{H}_{0}^{1}}+a(\mathbf{v}, \mathbf{w})-b(p, \mathbf{w}) & =\ell(\mathbf{w}), \\
b(q, \mathbf{v}) & =0 \\
(\mathbf{v}(\cdot, 0), \mathbf{w})_{0, \Gamma_{s}} & =(\hat{\mathbf{v}}, \mathbf{w})_{0, \Gamma_{s}} .
\end{aligned}
$$

Here, $a(\cdot, \cdot), b(\cdot, \cdot)$, and the functional $\ell(\cdot)$ are given by

$$
\begin{aligned}
a(\mathbf{v}, \mathbf{w}) & :=\left(\rho_{f}(\mathbf{v} \cdot \nabla) \mathbf{v}, \mathbf{w}\right)_{0, \Gamma_{s}}+(\eta \nabla \mathbf{v}, \nabla \mathbf{w})_{0, \Gamma_{s}} \\
b(p, \mathbf{v}) & :=(p, \nabla \cdot \mathbf{v})_{0, \Gamma_{s}}, \quad \ell(\mathbf{w}):=\left(\mathbf{f}_{q}, \mathbf{w}\right)_{0, \Gamma_{s}}+\left\langle\mathbf{f}_{g}, \mathbf{w}\right\rangle_{\mathbf{H}^{-1}, \mathbf{H}_{0}^{1}} .
\end{aligned}
$$

On the other hand, the weak formulation of (3.4) amounts to the computation of $\mathbf{X} \in \mathbf{Y}(0, T)$ such that for all $\mathbf{Z} \in \mathbf{H}_{\text {per }}^{3}([0, \ell])$ it holds

$$
\begin{aligned}
\left(\frac{d \mathbf{X}}{d t}(\cdot, t), \mathbf{Z}\right)_{0,(0, \ell)} & =(\mathbf{v}(\mathbf{X}(\cdot, t), t), \mathbf{Z})_{0,(0, \ell)}, \quad t \in[0, T] \\
(\mathbf{X}(\cdot, 0), \mathbf{Z})_{0,(0, \ell)} & =\left(\mathbf{X}^{0}, \mathbf{Z}\right)_{0,(0, \ell)} .
\end{aligned}
$$

4. The Backward Euler/Forward Euler FE-IB. For the discretization in space of the incompressible Navier-Stokes equations (3.9) we use Taylor-Hood P2/P1 elements with respect to a quasi-uniform simplicial triangulation $\mathcal{T}_{h}\left(\Gamma_{s}\right)$ of $\Gamma_{s}$. For $K \in \mathcal{T}_{h}\left(\Gamma_{s}\right)$, we denote by $|K|$ the area of $K$, by $h_{K}$ the diameter of $K$, and we set $h:=\max \left\{h_{K} \mid K \in \mathcal{T}_{h}\left(\Gamma_{s}\right)\right\}$. Further, $P_{k}(K), k \in \mathbb{N}$, refers to the set of polynomials of degree $\leq k$ on $K$. The associated finite element spaces $\mathbf{V}_{h}$ for the velocity and $Q_{h}$ for the pressure read

$$
\begin{aligned}
\mathbf{V}_{h} & :=\left\{\mathbf{v}_{h} \in \mathbf{C}\left(\bar{\Gamma}_{s}\right)\left|\mathbf{v}_{h}\right|_{K} \in P_{2}(K)^{2}, K \in \mathcal{T}_{h}\left(\Gamma_{s}\right),\left.\mathbf{v}_{h}\right|_{\partial \Gamma_{s}}=\hat{\mathbf{v}}_{h}\right\}, \\
Q_{h} & :=\left\{w_{h} \in C\left(\bar{\Gamma}_{s}\right)\left|w_{h}\right|_{K} \in P_{1}(K), K \in \mathcal{T}_{h}\left(\Gamma_{s}\right), \int_{\Gamma_{s}} w_{h} d x=0\right\}
\end{aligned}
$$


where $\hat{\mathbf{v}}_{h}$ is the $\mathbf{L}^{2}$-projection of $\hat{\mathbf{v}}$ onto the space of piecewise polynomials of degree 2 on $\partial \Gamma_{s}$. For the discretization of the boundary of the immersed enantiomer we consider a partition

$$
\mathcal{T}_{\Delta q}:=\left\{0=: q_{0}<q_{1}<\cdots<q_{R}:=\ell\right\}, R \in \mathbb{N},
$$

of the interval $[0, \ell]$ into subintervals $I_{i}:=\left[q_{r-1}, q_{r}\right], 1 \leq r \leq R$, of length $\Delta q_{r}:=$ $q_{r}-q_{r-1}$ with $\Delta q:=\max \left\{\Delta q_{r} \mid 1 \leq r \leq R\right\}$. We approximate $\mathbf{X}$ from (3.11) by periodic cubic splines

$$
\begin{aligned}
\mathbf{S}_{h}:=\left\{\mathbf{Z}_{h} \in \mathbf{C}^{2}([0, \ell]) \mid\right. & \left.\mathbf{Z}_{h}\right|_{I_{r}} \in P_{3}\left(I_{r}\right)^{2}, 1 \leq r \leq R, \\
& \left.\mathbf{Z}_{h}^{(k)}\left(q_{0}\right)=\mathbf{Z}_{h}^{(k)}\left(q_{R}\right), k=0,1,2\right\},
\end{aligned}
$$

where $P_{3}\left(I_{r}\right)$ stands for the set of polynomials of degree $\leq 3$ on $I_{r}$. For $\mathbf{Z}_{h} \in \mathbf{S}_{h}$, we set $\mathbf{Z}_{r}:=\mathbf{Z}_{h}\left(q_{r}\right), 0 \leq r \leq R$. The discrete immersed enantiomer occupies subdomains $B_{h, t} \subset \Gamma_{s}$ with boundaries $\partial B_{h, t}$ that are $C^{2}$ curves parameterized by the periodic cubic spline $\mathbf{X}_{h}(\cdot, t) \in \mathbf{S}_{h}$.

We define the total discrete energy by means of $E_{h}(t):=E_{h}^{e}(t)+E_{h}^{b}(t)$, where the discrete elastic energy $E_{h}^{e}(t)$ and the discrete bending energy $E_{h}^{b}(t)$ are given by

$$
\begin{aligned}
& E_{h}^{e}(t)=\frac{\kappa_{e}}{2} \int_{0}^{\ell}\left(\left|\frac{\partial \mathbf{X}_{h}}{\partial q}(q, t)\right|^{2}-1\right) d q, \\
& E_{h}^{b}(t)=\frac{\kappa_{b}}{2} \sum_{r=1}^{R} \int_{q_{r-1}}^{q_{r}}\left|\frac{\partial^{2} \mathbf{X}_{h}}{\partial q^{2}}(q, t)\right|^{2} d q .
\end{aligned}
$$

Observing that $\partial^{3} \mathbf{X}_{h}(q, t) / \partial q^{3}$ is constant on $I_{r}$, the discrete force density takes the form

$$
\begin{aligned}
& \left\langle\mathbf{f}_{h, g}(\cdot, t), \mathbf{w}_{h}\right\rangle_{h}=-\kappa_{e} \int_{0}^{\ell} \frac{\partial \mathbf{X}_{h}(q, t)}{\partial q} \cdot \frac{\partial}{\partial q} \mathbf{w}_{h}\left(\mathbf{X}_{h}(q, t)\right) d q \\
& +\kappa_{b} \sum_{i=1}^{R} \int_{q_{i-1}}^{q_{i}} \frac{\partial^{3} \mathbf{X}_{h}(q, t)}{\partial q^{3}} \cdot \frac{\partial}{\partial q} \mathbf{w}_{h}\left(\mathbf{X}_{h}(q, t)\right) d q= \\
& -\kappa_{e} \int_{0}^{\ell} \frac{\partial \mathbf{X}_{h}(q, t)}{\partial q} \cdot \nabla \mathbf{w}_{h}\left(\mathbf{X}_{h}(q, t)\right) \frac{\partial \mathbf{X}_{h}(q, t)}{\partial q} d q \\
& +\left.\kappa_{b} \sum_{r=1}^{R} \frac{\partial^{3} \mathbf{X}_{h}(q, t)}{\partial q^{3}}\right|_{I_{r}} \cdot \int_{q_{r-1}}^{q_{r}} \nabla \mathbf{w}_{h}\left(\mathbf{X}_{h}(q, t)\right) \frac{\partial \mathbf{X}_{h}(q, t)}{\partial q} d q .
\end{aligned}
$$

We thus obtain the following approximation of the right-hand side in (3.9a)

$$
\ell_{h}\left(\mathbf{w}_{h}, t\right):=\left(\mathbf{f}_{q}, \mathbf{w}_{h}\right)_{0,(0, \ell)}+\left\langle\mathbf{f}_{h, g}(\cdot, t), \mathbf{w}_{h}\right\rangle_{h} .
$$

The discretization in time is done with respect to an equidistant partition

$$
\mathcal{T}_{\Delta t}:=\left\{0=: t_{0}<t_{1}<\cdots<t_{M}:=T\right\}, M \in \mathbb{N},
$$


of the time interval $[0, T]$ into subintervals of length $\Delta t:=T / M$. We denote by $\mathbf{v}_{h}^{(m)}$ an approximation of $\mathbf{v}_{h} \in \mathbf{V}_{h}$ at $t=t_{m}$. We further refer to $\mathbf{D}_{\Delta t}^{+} \mathbf{v}_{h}^{(m)}:=$ $\left(\mathbf{v}_{h}^{(m+1)}-\mathbf{v}_{h}^{(m)}\right) /(\Delta t)$ and $\mathbf{D}_{\Delta t}^{-} \mathbf{v}_{h}^{(m)}:=\left(\mathbf{v}_{h}^{(m)}-\mathbf{v}_{h}^{(m-1)}\right) /(\Delta t)$ as the forward and backward difference operator. We set

$$
\begin{aligned}
& \mathbf{W}_{h}^{(m)}:=\left\{\mathbf{w}_{h}^{(m)} \in \mathbf{C}\left(\bar{\Gamma}_{s}\right)\left|\mathbf{w}_{h}^{(m)} \in \mathbf{V}_{h}, \mathbf{w}_{h}^{(m)}\right|_{\partial \Gamma_{s}}=\hat{\mathbf{v}}_{h}\right\}, \\
& Q_{h}^{(m)}:=\left\{w_{h}^{(m)} \in C\left(\bar{\Gamma}_{s}\right)\left|w_{h}^{(m)}\right|_{K} \in Q_{h}, \int_{\Gamma_{s}} w_{h}^{(m)} d x=0\right\} .
\end{aligned}
$$

The Backward Euler/Forward Euler FE-IB reads as follows: Given $\mathbf{v}_{h}^{(0)}=\hat{\mathbf{v}}_{h}$ and $\mathbf{X}_{h}^{(0)} \in \mathbf{S}_{h}$, for $m=0, \ldots, M-1$ we perform the following two steps:

Step 1: Compute $\left(\mathbf{v}_{h}^{(m+1)}, p_{h}^{(m+1)}\right) \in \mathbf{W}_{h}^{(m+1)} \times Q_{h}^{(m+1)}$ such that for all $\mathbf{w}_{h} \in$ $\mathbf{V}_{h, 0}:=\left\{\mathbf{w}_{h} \in \mathbf{V}_{h}\left|\mathbf{w}_{h}\right|_{\partial \Gamma_{s}}=\mathbf{0}\right\}$ it holds

$$
\begin{aligned}
\left(\rho_{f} \mathbf{D}_{\Delta t}^{+} \mathbf{v}_{h}^{(m)}, \mathbf{w}_{h}\right)_{0, \Gamma_{s}}+a\left(\mathbf{v}_{h}^{(m+1)}, \mathbf{w}_{h}\right)-b\left(p_{h}^{(m+1)}, \mathbf{w}_{h}\right) & =\ell_{h}^{(m)}\left(\mathbf{w}_{h}\right), \\
b\left(w_{h}, \mathbf{v}_{h}^{(m+1)}\right) & =0
\end{aligned}
$$

where $\ell_{h}^{(m)}\left(\mathbf{w}_{h}\right):=\ell_{h}\left(\mathbf{w}_{h}, t_{m}\right)$ is given by (4.3).

Step 2: Compute $\mathbf{X}_{h}^{(m+1)} \in \mathbf{S}_{h}$ such that for all $\mathbf{Z}_{h} \in \mathbf{S}_{h}$ it holds

$$
\left(\mathbf{D}_{\Delta t}^{+} \mathbf{X}_{r}^{(m)}, \mathbf{Z}_{h}\right)_{0,(0, \ell)}=\left(\mathbf{v}_{h}^{(m+1)}\left(\mathbf{X}_{r}^{(m)}\right), \mathbf{Z}_{h}\right)_{0,(0, \ell)}, \quad 1 \leq r \leq R .
$$

As has been shown in [14, 18], the Backward Euler/Forward Euler FE-IB is not unconditionally stable, but has to satisfy the CFL-type stability condition

$$
\frac{\Delta t}{h} \leq \frac{\eta}{4 C\left(\kappa_{e} L_{1}+\kappa_{b} L_{2}\right)},
$$

where $C>0$ is a constant depending on the size and shape of the immersed enantiomer and $L_{1}, L_{2}$ are given by

$$
L_{1}:=\max _{0 \leq m \leq M} \max _{q \in[0, \ell]}\left|\frac{\partial \mathbf{X}_{h}^{(m)}}{\partial q}\right|, \quad L_{2} .=\max _{0 \leq m \leq M} \max _{1 \leq r \leq R}\left|\frac{\partial^{3} \mathbf{X}_{h}^{(m)}}{\partial q^{3}}\right|_{I_{r}} \mid .
$$

5. Numerical results. We present the results of numerical simulations of the separation of deformable, initially L-shaped enantiomers by SAW generated vorticity patterns consisting of four pairwise counter-rotating vortices at the surface of the fluid. The material data and the numerical data have been chosen as follows:

Material Data: As piezoelectric material we have used $128^{\circ}$ rotated YX lithium niobate $\left(\mathrm{LiNbO}_{3}\right)$ with density $\rho_{p}=4.63 \cdot 10^{3} \mathrm{~kg} / \mathrm{m}^{3}$ and elasticity tensor c, piezoelectric tensor $\mathbf{e}$, and dielectric tensor $\boldsymbol{\epsilon}$ given in Table 5.1. The operating frequency $f$ of the IDT has been chosen according to $f=1.42 \cdot 10^{2} \mathrm{MHz}$. It turns out that $v_{0}(f)=2.0 \cdot 10^{-3} \mathrm{~m} / \mathrm{s}$ in $(3.3)$ provides a good approximation of the resulting velocity pattern at the surface of the fluid.

The fluid with density $\rho_{f}=1.1 \cdot 10^{3} \mathrm{~kg} / \mathrm{m}^{3}$ and viscosities $\eta=\xi=1.01 \cdot 10^{-6} \mathrm{~m}^{2} / \mathrm{s}$ occupied a domain $D=(0, L)^{2} \times(0, H)$ with $L=4.0 \cdot 10^{-2} \mathrm{~m}$ and $H=5.0 \cdot 10^{-3}$ 
TABLE 5.1

Material Moduli for $128^{\circ}$ rotated $\mathbf{Y X ~} \mathrm{LiNbO}_{3}$ (note that $c_{11}=c_{22}, c_{13}=c_{23}, c_{14}=-c_{24}=$ $c_{56}, c_{44}=c_{55}$ and $e_{22}=-e_{16}$ )

\begin{tabular}{|c|c|c|c|c|c|c|c|}
\hline$\left[10^{10} \frac{\mathbf{c}}{\mathbf{m}^{2}}\right]$ & $\begin{array}{c}c_{11} \\
20.3\end{array}$ & $\begin{array}{l}c_{12} \\
5.3 \\
\end{array}$ & $\begin{array}{l}c_{13} \\
7.5 \\
\end{array}$ & $\begin{array}{l}c_{14} \\
0.9\end{array}$ & $\begin{array}{c}c_{33} \\
24.5\end{array}$ & $\begin{array}{l}c_{44} \\
6.0\end{array}$ & $\begin{array}{l}c_{66} \\
7.5\end{array}$ \\
\hline $\begin{array}{c}\mathbf{e} \\
{\left[\frac{\mathbf{C}}{\mathbf{m}^{2}}\right]}\end{array}$ & \multicolumn{2}{|c|}{$\begin{array}{c}e_{15}=e_{24} \\
3.7\end{array}$} & \multicolumn{2}{|c|}{$\begin{array}{c}e_{22}=-e_{21} \\
2.5\end{array}$} & \multicolumn{2}{|c|}{$\begin{array}{c}e_{31}=e_{32} \\
0.1\end{array}$} & $\begin{array}{l}e_{33} \\
1.3 \\
\end{array}$ \\
\hline $\begin{array}{c}\boldsymbol{\epsilon} \\
{\left[10^{-12} \frac{\mathbf{F}}{\mathbf{m}}\right]}\end{array}$ & \multicolumn{4}{|c|}{$\begin{array}{c}\epsilon_{11}=\epsilon_{22} \\
749.0\end{array}$} & \multicolumn{3}{|c|}{$\begin{array}{c}\epsilon_{33} \\
253.2\end{array}$} \\
\hline
\end{tabular}

$\mathrm{m}$. The material moduli of the enantiomers were given by $\kappa_{e}=3 \cdot 0 \cdot 10^{-2} \mathrm{~N} / \mathrm{m}$ and $\kappa_{b}=2 \cdot 5 \cdot 10^{-17} \mathrm{Nm}$.

Numerical Data: For the numerical solution of the coupled system (3.9),(3.11) by the Backward Euler/Forward Euler FE-IB we have used a uniform simplicial triangulation of $\Gamma_{s}$ by right isosceles with $h=L / 35$ and a uniform partition of $[0, \ell]$ with $\Delta q=1 / 500$. We have further used a uniform partition of the time interval with time step size $\Delta t=1 / 2000$ satisfying the CFL-condition (4.6). All computations have been performed under Linux featuring Intel(R)Core(TM) i3-2100 CPU $3.10 \mathrm{GHz}$ and 7.7 GB RAM.

Simulation Results: An initially L-shaped, left-handed enantiomer has been injected onto the surface of the fluid approximately in the middle between two counterrotating vortices. The motion of the enantiomer is such that it gets attracted by the right-rotating fluid vortex. After the completion of the first cycle around the center of the vortex, new cycles begin with pathes of the enantiomer similar to the first one (cf. Figure 5.1). Figure 5.2 displays the pathes of an initially L-shaped, right-handed enantiomer which gets attracted by the left-rotating vortex. The separation does not depend on the position of the enantiomers with respect to the velocity field (see Figure 5.3 for a left-handed L-shaped enantiomer rotated by $90^{\circ}$ compared to the upright position). However, for a proper separation of the enantiomers it is important that they are injected approximately in the middle between two counter-rotating vortices. 

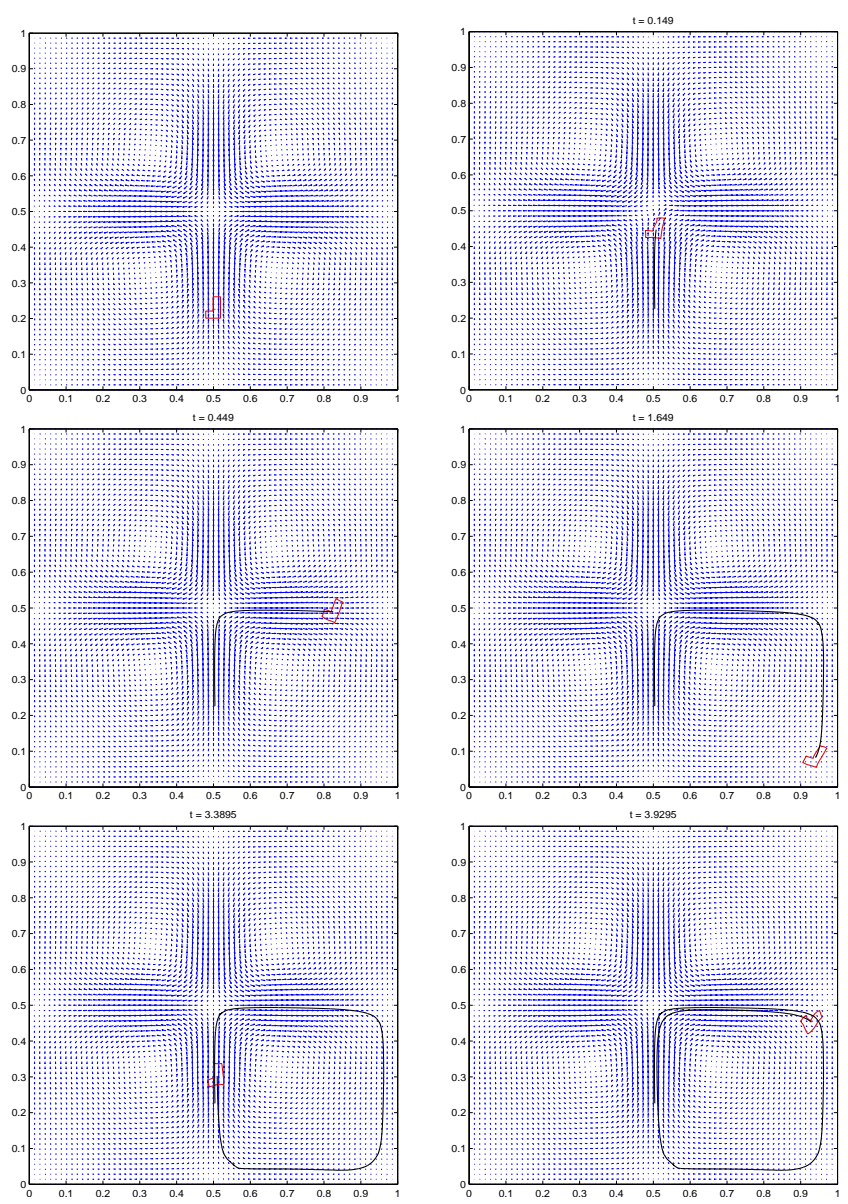

FIG. 5.1. Velocity field and motion of a left-handed L-shaped enantiomer initially placed in the middle between two counter-rotating vortices

\section{REFERENCES}

[1] H. Antil, R. Glowinski, R. H. W. Hoppe, C. Linsenmann, T.-W. Pan, and A. Wixforth; Modeling, simulation, and optimization of surface acoustic wave driven microfluidic biochips. J. Comp. Math. 28, 149-169, 2010.

[2] K. Bester; Chiral analysis for environmental applications. Anal. Bioanal. Chem. 376, 302-304, 2003.

[3] J.-B. Biot; Bull. Soc. Philomath. 6, 190-192, 1815

[4] D. Boffi and L. Gastaldi; A finite element approach for the immersed boundary method. Comput. Struct. 81, 491-501, 2003.

[5] D. Boffi, L. Gastaldi, and L. Heltai; Numerical stability of the finite element immersed boundary method. Math. Models and Methods in Appl. Sci. 17, 1479-1505, 2007.

[6] M. Born; Electron theory of natural optic rotation processes in isotropic and anisotropic liquids. Ann. Phys. 55, 177-240, 1918.

[7] K.A. Busch and M.A. Busch (eds.); Chiral Analysis. Elsevier, Amsterdam, 2006.

[8] A.N. Collins, G. Sheldrake, and J. Crosby (eds.); Chirality in Industry: The Commerical Manufacture and Applications of Optically Active Compounds. Wiley, Chichester, 1995. 

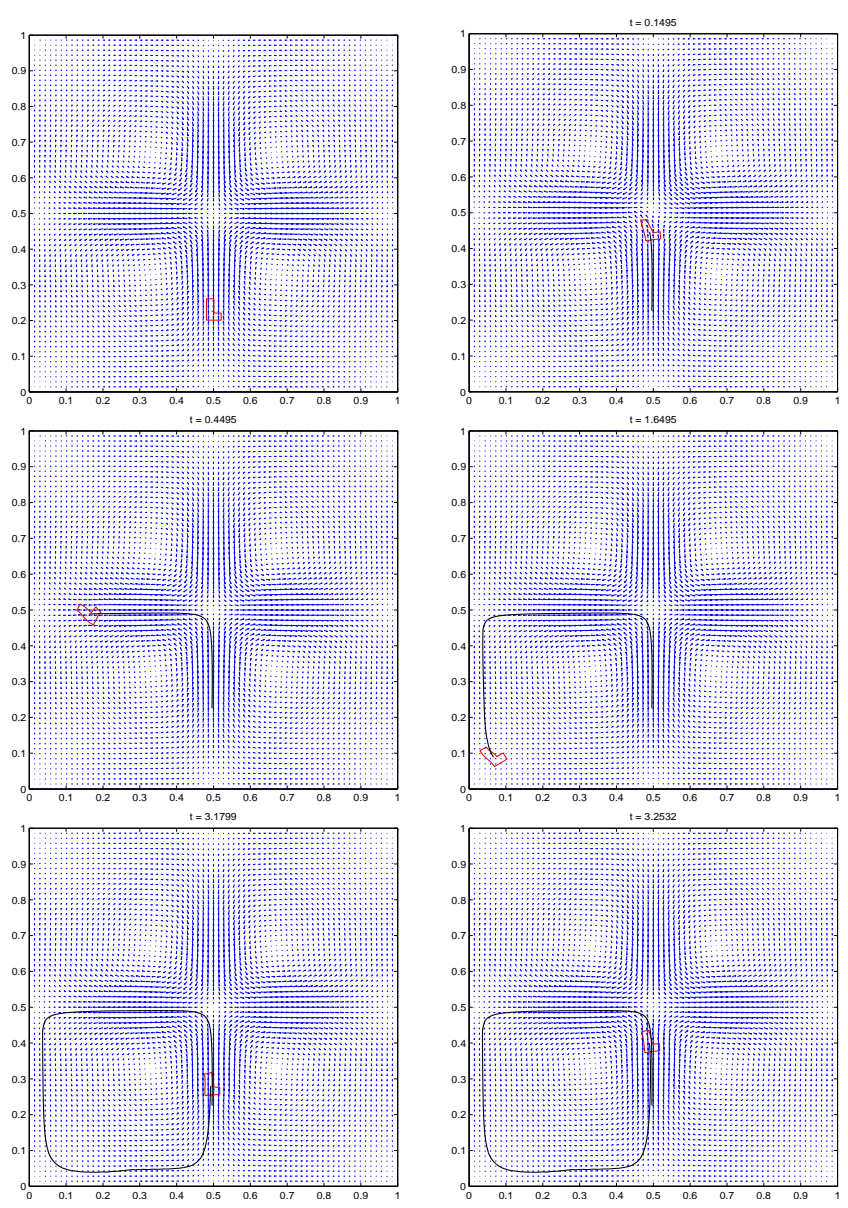

FIG. 5.2. Velocity field and motion of a right-handed L-shaped enantiomer initially placed in the middle between two counter-rotating vortices

[9] A.N. Collins, G. Sheldrake, and J. Crosby (eds.); Chirality in Industry II: The Commerical Manufacture and Applications of Optically Active Compounds. Wiley, Chichester, 1997.

[10] E.U. Condon, W. Altar, and H. Eyring; One electron rotatory power. J. Chem. Phys. 5, 479-491, 1937.

[11] M. Eisenstein; Cell sorting: divide and conquer, Nature 441, 1179-1185, 2006.

[12] T. M. Fischer, M. Stohr-Liesen, and Schmid-Schönbein; Science 202, 894, 1978.

[13] E. Francotte and W. Lindner (eds.); Chirality in Drug Research. Wiley-VCH, Weinheim, 2006.

[14] T. Franke, R.H.W. Hoppe, C. Linsenmann, L. Schmid, and C. Willbold; Numerical simulation of the motion and deformation of red blood cells and vesicles in microfluidic flows. Comput. Visualiz. Sci. 14, 167-180, 2011.

[15] J. Gal; The discovery of biological enantioselectivity: Louis Pasteur and the fermentation of tartaric acid - a review and analysis $150 \mathrm{yr}$ later. Chirality 20, 5-19, 2008.

[16] A. Gantner, R.H.W. Hoppe, D. Köster, K.G. Siebert, and A. Wixforth; Numerical simulation of piezoelectrically agitated surface acoustic waves on microfluidic biochips. Comp. Visual. Sci. 10, 145-161, 2007. 

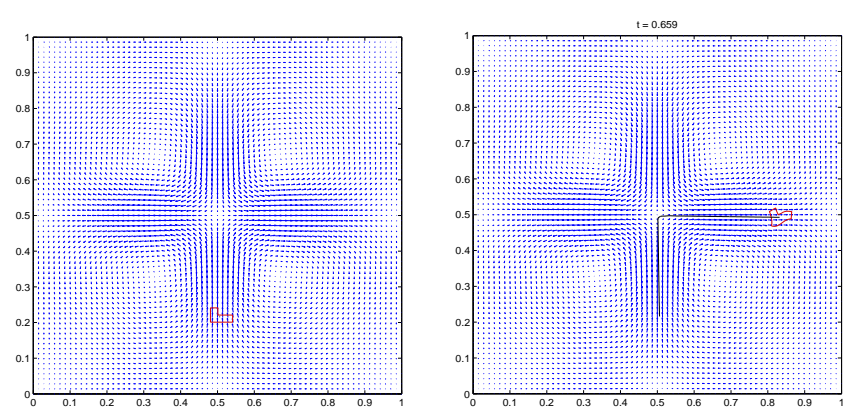

FIG. 5.3. Velocity field and motion of a left-handed L-shaped enantiomer rotated by $90^{\circ}$ with respect to the upright position

[17] J.H. van't Hoff; Utrecht, Netherlands, 1874.

[18] R.H.W. Hoppe and C. Linsenmann; An adaptive Newton continuation strategy for the fully implicit finite element immersed boundary method. J. Comp. Phys. 231, 4676-4693, 2012.

[19] F. Hund; Z. f. Phys. 43, 806, 1927.

[20] D. Köster; Numerical simulation of acoustic streaming on SAW-driven biochips. SIAM J. Comp. Sci., 29, 2352-2380, 2007.

[21] M. Konrad; Trennung chiraler Objekte in Mikroströmung, Diploma Thesis, University of Augsburg, 2008.

[22] M. Kostur, M. Schindler, P. Talkner, and P. Hänggi; Chiral separation in microflows. Phys. Rev. Lett. 96, 014502-1 - 014502-4, 2006.

[23] N. Kurihara and J. Miyamoto (eds.); Chirality in Agrochemicals. Wiley, Chichester, 1998.

[24] J.A. LeBel; Bull. Soc. Chim. 22, 337-347, 1874.

[25] P.C.H. Li; Microfluidic Lab-on-a-Chip for Chemical and Biological Analysis and Discovery. CRC Press, Boca Raton, 2006.

[26] Marcos, H.C. Fu, T.R. Powers, and R. Stocker; Separation of microscale chiral objects by shear flow. Phys. Rev. Lett. 102, 158103-1 - 158103-4, 2009.

[27] L. Pasteur; Compt. Rend. Acad. Sci. 26, 535-538, 1848.

[28] L. Pasteur; Compt. Rend. Acad. Sci. 37, 110-114, 1853.

[29] R.N. Patel (ed.); Stereoselective Biocatalysis. CRC Press, Boca Raton, 2000.

[30] C. Peskin; Numerical analysis of flood flow in the heart. J. Comput. Phys. 25, 220-252, 1977

[31] C.S. Peskin; The immersed boundary method. Acta Numerica 11, 479-517, 2002.

[32] M. Quack and J. Strohner; Parity violation in chiral molecules. Chimia 59, 530-538, 2005.

[33] M. Quack, J. Strohner, and M. Willeke; High-resolution spectroscopic studies and theory of parity violation in chiral molecules. Ann. Rev. Phys. Chem. 59, 741-769, 2008.

[34] G.K.E.V. Scriba; Cyclodextrins in capillary electrophoresis enantioseparations - Recent developments and applications. J. Sep. Sci. 31, 1991-2011, 2008.

[35] P. Talkner, G. Ingold, and P. Hänggi; Transport of flexible chiral objects in a uniform shear flow. New Journal of Physics 14, 073006, 2012.

[36] L. Tartar; Introduction to Sobolev Spaces and Interpolation Theory. Springer, BerlinHeidelberg-New York, 2007.

[37] C.J. Welch; Microscale chiral HPLC in support of pharmaceutical process research. Chirality 21, 114-118, 2009.

[38] T. J. Wenzel; Discrimination of Chiral Compounds using NMR Spectroscopy. Wiley, Chichester, 2007. 